\title{
Radioisotope scanning of the lungs
}

\author{
T. K. BELL, R. FERGUSON, E. I. MCILRATH, AND \\ J. A. WEAVER \\ From the Royal Victoria Hospital, Belfast 12, Northern Ireland
}

\begin{abstract}
Lung scanning with macroaggregated albumin ${ }^{131} \mathrm{I}$ was carried out in 128 patients. The technique appears to be without hazard. It is particularly useful in the detection of pulmonary embolism, but the pattern is non-specific and changes occur in other cardio-respiratory diseases. On the basis of these observations pulmonary scanning is regarded as a useful adjunct in the study of pulmonary embolism, but it cannot serve as a specific diagnostic procedure since similar patterns of scan may be found in many conditions.
\end{abstract}

The diagnosis of pulmonary embolus is difficult to make in many instances, yet the need for a greater degree of certainty is desirable, first in order to treat adequately the individual patient and, more generally, to assess the value of the various means of treatment, either embolectomy or anticoagulant treatment. The causes of the difficulty in diagnosis may be summarized as follows. The clinical findings are diverse (Gorham, $1961 \mathrm{a}, \mathrm{b}, \mathrm{c})$; the classical electrocardiographic pattern (McGinn and White, 1935) occurs in only a proportion of patients; and blood enzyme studies based on a specific rise in the lactic dehydrogenase level (Wacker and Snodgrass, 1960) have been found to be unreliable (Schonell, Crompton, Forshall, and Whitby, 1966). A plain chest radiograph interpreted according to the criteria of Fleischner (1962) and Chrispin, Goodwin, and Steiner (1963) is often helpful ; but the changes may be either absent or present to a limited degree. Pulmonary angiography provides the most satisfactory evidence, but the procedure is not without risk in this group of patients.

Scintillation scanning of the lungs has been advocated (Wagner, Sabiston, McAfee, Tow, and Stern, 1964), but the reliability and specificity of this test have been questioned (Fred, Burdine, Gonzalez, Lockhart, Peabody, and Alexander, 1966). This paper reports the findings in 128 pulmonary scans with ${ }^{131}$ I-labelled macroaggregated albumin, and in 25 instances a comparison was made between the isotope scan and the pulmonary angiographic appearances.

\section{GENERAL PRINCIPLES}

The original observations of Taplin, Johnson, Dore, and Kaplan (1964) and of Wagner et al.
(1964) established the safety and principles of the clinical method. It had been demonstrated previously that aggregates of human serum albumin could be made by alterations in the $p H$ and temperature of the albumin solution. By these techniques a macroaggregated albumin, particle size 1 to 100 microns, was achieved, the majority of particles being in the range of 10 to 100 microns. Following intravenous injection the particles are trapped in the pulmonary capillaries during the initial transit of the lung fields, and the distribution in any segment of the lung is proportional to the relative pulmonary blood flow. Various radioactive labels of the albumin aggregates have been used- ${ }^{51} \mathrm{Cr},{ }^{131} \mathrm{I}$, and ${ }^{99 \mathrm{~m}} \mathrm{Tc}$. The albumin aggregates are readily metabolized and the rate of clearance from the lung fields has a half-life ranging between five and 10 hours.

The inherent dangers of the procedure are allergic reactions to the albumin, infection of the albumin solution prior to injection, haemodynamic changes secondary to the diffuse micro-embolization of the pulmonary vascular bed, and the radiation dosage from the isotope.

\section{MATERIAL AND METHODS}

PATIENTS At the time of performing the pulmonary scans a definitive diagnosis had not been established in many instances. The diagnosis of thromboembolism was eventually accepted from the clinical course of the patient, particularly the response to heparin therapy, electrocardiographic findings, serial chest films, enzyme studies (transaminase and lactic dehydrogenase), and pulmonary angiography. Pulmonary angiography was undertaken if there was reasonable doubt regarding the other criteria and if the patient was considered to be sufficiently fit to stand the examination without undue risk. 
Usually, the sequence of events was to do the pulmonary scan as soon as possible and to perform the pulmonary angiogram 24 hours later, but in some instances the delay was 48 to 72 hours.

\section{TECHNIQUE}

Pulmonary scanning Prior to lung scanning, patients were started on Lugol's iodine orally, which was continued for three to four days. The patient was given 200 to $275 \mu \mathrm{Ci}{ }^{131} \mathrm{I}$ macroaggregated human serum albumin by intravenous injection over a period of two minutes while lying in the supine position and breathing deeply, scanning being started 10 to 30 minutes later.

An I.D.L. ${ }^{1}$ body scanner incorporating colour printout was used. In the early part of the series one $19-$ hole $16-\mathrm{cm}$. focusing collimator was used in association with a 3 -in. diameter 2 -in. thick sodium iodide crystal for anterior scans with the patient lying in the supine position. This system gave a limited view of the lung fields as shown by the difference between anterior and posterior scans. To increase the field of view through the lungs in the latter part of the series two $16-\mathrm{cm}$. focusing collimators in axial alignment were used. One was placed just below the couch top. which moved relative to the detectors, and the other 32 to $34 \mathrm{~cm}$. from it above the couch top. Both detecting systems were matched for equal sensitivity using pulse height analysis to accept pulses corresponding to gamma radiation in the energy band 0.31 to 0.41 $\mathrm{MeV}$, and the scan picture was produced from the sum of the pulses from each detector.

The maximum observed count rate over the lungs varied between 7,200 and 18,000 per minute for the single collimator system and between 18,000 and 48,000 per minute for the double collimator system. The scanning speeds varied between 25 and $45 \mathrm{~cm}$. per minute, and 40 to 75 minutes were required to complete a scan.

Two commercial preparations of ${ }^{131}$ I macroaggregated human serum albumin were obtained for the investigations: from Squibb, U.S.A., with a specific activity on receipt of approximately 0.39 $\mathrm{mCi} / \mathrm{mg}$. albumin; and from Sorin, Italy, with a specific activity of 0.11 to $0.18 \mathrm{mCi} / \mathrm{mg}$. albumin. Some batches of the latter product appeared to be removed from the lung fields rapidly with a half-life of only 90 minutes compared with the Squibb product, which had a half-life in the lung fields of over five hours. (A scan of one patient four hours after injection of the Sorin macroaggregated human serum albumin showed a large distribution of radioactivity in the liver and a count rate over the lungs about one-fifth of the initial activity immediately after injection.) Investigations of the Sorin particles under the microscope showed $80 \%$ to be needle-shaped, of diameter approximately 7 to 20 microns and length 10 to 60 microns, compared with the Squibb particles,

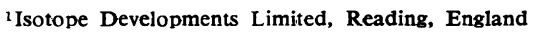

of which $80 \%$ were irregular square-shaped of size 10 to 60 microns.

The maximum amount of albumin aggregates injected was $8 \mathrm{mg}$. of the Sorin product due to the lower specific activity compared with $2 \mathrm{mg}$. of the Squibb product. When not in use the preparations were stored at $4^{\circ} \mathrm{C}$., and two weeks after receipt analysis of the remainder showed 17 to $29 \%$ and $14 \%$ inorganic iodine in the Squibb and Sorin preparations respectively.

Pulmonary angiography The factors governing the choice of technique were unavailability of physiological monitoring equipment (including electrocardiograph), which precluded selective injection into the main pulmonary artery and its branches, and the desire to test a simple technique which any member of a radiological staff would carry out. For these reasons it was decided that the percutaneous Seldinger technique using local anaesthesia was ideal, and whenever possible the left median antecubital vein was used. After initial trials it was found that the most suitable catheter was a No. 6 or No. 8 Gensini Tefion catheter introduced on a P.E.160 Teflon-coated guide wire. The catheter was sited in the lower superior vena cava, and sodium metrizoate $75 \%$ (Triosil ' 75 ') was injected by Gidlund pressure injector at 5 to 6 $\mathrm{kg} . / \mathrm{cm} .{ }^{2}$ in a dose of $1 \mathrm{cc} / \mathrm{kg}$. body weight. Anteroposterior films were then taken at intervals of 1.5 seconds for 10 seconds, the first exposure being automatically triggered after $10 \mathrm{cc}$. of contrast medium had been injected. If the standard chest radiograph suggested pulmonary hypertension, the film programme was delayed until at least half the contrast medium had been injected. The only absolute contraindication recognized was the recent onset of a cardiac arrhythmia. Relative contraindications included severe pulmonary hypertension and cor pulmonale or a history of a reaction to contrast media.

The criteria for the angiographic diagnosis of pulmonary embolism were (1) the demonstration of a filling defect in a pulmonary artery; (2) the abrupt termination of a vessel of prelobular size or larger ; and (3) the presence of an area of oligaemia with delayed or absent venous filling if allied to signs 1 or 2 (Abrams, 1961).

\section{RESULTS}

We have performed 128 pulmonary scans (the discrepancy in the totals in the tables is due to some patients having had repeat scans performed). In the tables the results are tabulated as positive or negative, positive meaning a definite area of diminished or absent radioactivity, indicating a decrease in pulmonary perfusion of that zone. Table I shows the results in normal patients and in a variety of disease states which might be expected to alter pulmonary blood flow. 
T A B L E I

THE PATTERN OF PULMONARY SCAN IN 108 PATIENTS

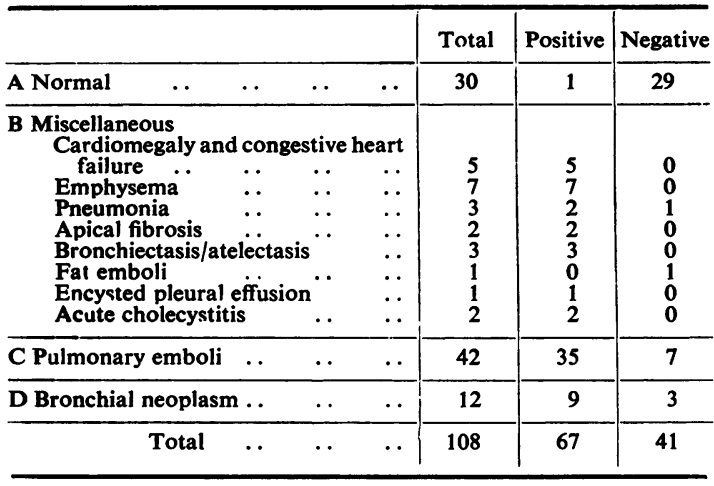

In 29 instances out of 30 in normal individuals pulmonary scanning produced a pattern of the lung fields that seemed normal in respect of the size of the lungs and the contours of the lung margins when contrasted with the plain chest radiograph. In the remaining patient an abnormal scan showing avascularity of the right mid-zone was not confirmed by an angiographic study.

In the various disease states the distortion of the pulmonary vascular pattern was considerable. Cardiomegaly per se decreased radioactivity at the left base, and, when associated with congestive heart failure. diminished activity at both bases was noted. Emphysema, usually with discernible bullae, caused marked changes, as did apical fibrosis and basal bronchiectasis.

The two patients with acute cholecystitis were strikingly similar in that right hypochondriac and lower right chest discomfort were the complaints ; in both instances elevation of the right dome of the diaphragm was demonstrated and the scan showed diminished filling at the right base.

Pulmonary embolic disease was correctly demonstrated by the scan in 35 of 42 instances (Table $\mathrm{I}(\mathrm{C})$ ). The presumed causes of failure to demonstrate the embolic lesion in seven patients are listed in Table II. In four of these patients the definitive diagnosis of embolism had been confirmed by pulmonary angiography, but in the other three the diagnosis of embolism, though strongly supported by clinical and plain radiological findings, can only be regarded as tentative.

It will be seen that the failure to demonstrate embolic lesions was due either to the inability to losalize minor emboli, particularly in the basal zones, or to a delay in performing the scan. It was apparent by performing serial scans on some patients that recanalization or dissolution of the emboli is rapid and that a scan performed as soon as four to five days after the embolic event may already have reverted to normal. Experimental work by Wagner et al. (1964) has demonstrated the rapidity of resolution of experimental thrombo-embolic lesions in animals, and in a recent study (Tow and Wagner, 1967) it has been

T A B L E I I

SUSPECTED CAUSES OF NEGATIVE SCANS IN PULMONARY EMBOLIC DISEASE (7 PATIENTS)

\begin{tabular}{|c|c|c|c|c|}
\hline Case & $\begin{array}{c}\text { Sex and } \\
\text { Age }\end{array}$ & Embolus & Angiography & $\begin{array}{c}\text { Cause of } \\
\text { Negative Scan }\end{array}$ \\
\hline 1 & M 47 & Right base & - & $\begin{array}{l}\text { Small right basal } \\
\text { embolus; also } \\
\text { delay in perform- } \\
\text { ing scan (fourth } \\
\text { day) }\end{array}$ \\
\hline 2 & F 25 & $\begin{array}{l}\text { Left lower } \\
\text { base }\end{array}$ & $\begin{array}{l}\text { Occlusion of seg- } \\
\text { mental artery } \\
\text { retrocardiac zone }\end{array}$ & $\begin{array}{l}\text { Lesion within car- } \\
\text { diac area of scan }\end{array}$ \\
\hline 3 & M 62 & Right base & Ietrocar urac zoric & $\begin{array}{l}\text { ? delay in perform- } \\
\text { ing scan (fifth } \\
\text { day) }\end{array}$ \\
\hline 4 & M 66 & $\begin{array}{l}\text { Repeated } \\
\text { small } \\
\text { emboli }\end{array}$ & $\begin{array}{l}\text { Multiple pruning } \\
\text { of small vessels }\end{array}$ & $\begin{array}{l}\text { Symmetrical nature } \\
\text { of defect involv- } \\
\text { ing small vessels }\end{array}$ \\
\hline 5 & F 58 & Left base & - & $\begin{array}{l}\text { Retrocardiac area; } \\
\text { also some car- } \\
\text { diac enlargement }\end{array}$ \\
\hline 6 & F 44 & $\begin{array}{r}\text { Repeated } \\
\text { emboli }\end{array}$ & $\begin{array}{l}\text { Several basal ar- } \\
\text { teries occluded } \\
\text { (both bases) }\end{array}$ & $\begin{array}{l}\text { Symmetrical nature } \\
\text { of defect involv- } \\
\text { ing small vessels }\end{array}$ \\
\hline 7 & F 56 & $\begin{array}{r}\text { Repeated } \\
\text { emboli }\end{array}$ & $\begin{array}{l}\text { Several small basal } \\
\text { segmental arteries } \\
\text { occluded }\end{array}$ & $\begin{array}{l}\text { Symmetrical nature } \\
\text { of defect involv- } \\
\text { ing small vessels }\end{array}$ \\
\hline
\end{tabular}

shown that human thrombo-embolic disease is usually followed by rapid recovery of pulmonary arterial blood flow.

The converse of this situation was observed in two patients in this series who had abnormal pulmonary scans at six to nine months after major pulmonary emboli. In one of these it was found that she had arterial oxygen desaturation and poly- 윽 cythaemia, and pulmonary angiography confirmed $D$ the scan findings of avascularity to a major portion of the right lower lobe.

The major difficulty in detecting minor emboli o is their localization to the peripheral basal zones. $N$ It is in these areas that it is difficult to decide $\underset{\omega}{N}$ whether : a scan is abnormal or not. In normalo patients lying in the supine position there is con-co siderable variation of the appearance of the right $\stackrel{\varnothing}{\mathbb{D}}$ base, and in some scans the right lung field is $\stackrel{\infty}{+}$ foreshortened to some degree. Injection of the $T$ isotope was always carried out in the supine position to ensure filling of the upper lung zones; it seems possible that the recumbent position $\stackrel{\mathbb{Q}}{\mathscr{Q}}$ allows the liver to exert some pressure on the 
retrohepatic zone of the right lung base and that filling of this zone is consequently diminished. A similar effect occurs also at the left lung base, but is confined to the retrocardiac zone, and the foreshortening does not extend to the costophrenic angle.

In one patient (case 2, Table II) a pulmonary angiographic study disclosed embolization of a left lower lobe retrocardiac branch, but it was impossible to distinguish any abnormality on the scan. Fred et al. (1966) have emphasized the difficulty in interpreting changes in the lower zones; they found that patients with failure of the left ventricle had diminished radioactivity over one or both lower lung fields. These workers also reported that cardiac enlargement would affect vascular flow to the left base, and in the present series one patient with severe hypertension and cardiomegaly not in cardiac failure had a markedly diminished radioactivity at the-left base.

Pulmonary angiography was carried out in 25 patients and the examination was successful in all but two. In one patient the examination was terminated when a catheter could not be passed more than $4 \mathrm{~cm}$. proximally in any of the arm veins. In the second patient the films were unsatisfactory due to failure to place the catheter in the superior vena cava, with resultant poor filling of the pulmonary circulation. In a third patient the only potentially serious complication occurred. A P.E.240 Clay Adams polyethylene catheter was placed low in the superior vena cava. The patient complained of severe substernal pain on injection of the contrast, and it was found that the catheter had recoiled into the azygos vein, with extravasation of contrast into the mediastinum. The films, although of poor quality, were considered diagnostic and the patient recovered completely within 24 hours. Recoil would probably not have occurred if the more rigid Teflon catheter had been used.

Table III summarizes the results obtained in 25 pulmonary angiograms. The correlation between pulmonary scanning and pulmonary angiography as regards the site and extent of embolic disease was excellent in 13 patients. The presumed cause of discrepancy in the four patients who had both angiography and scanning performed is referred to in Table II.

A fifth case is of interest. Both the scan and the angiogram were abnormal but there was a discrepancy between them with regard to localization of the lesions. The patient had a history suggestive of recurrent multiple small emboli. The scan was abnormal, showing a shortening of both
T A B L E I I I

PULMONARY ANGIOGRAMS AND SCANS

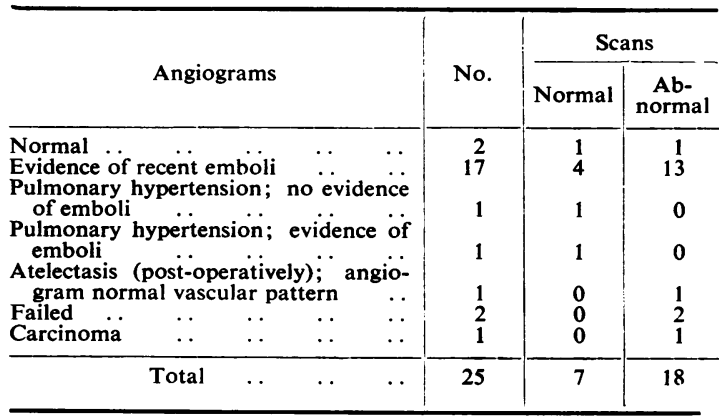

lung fields, in that both bases appeared to have equally negative basal zones but the angiogram showed blockage of a single prelobular artery to the right base, whereas on the left no abnormality was discernible except delay in the venous filling phase in the left mid-zone.

In the series of patients we have studied, no untoward symptom or reaction occurred following injection of the albumin aggregates. The coloured illustrations show some typical scans in a variety of conditions. The coloured areas represent radioactivity in the following proportional scale: blue, $100-85 \%$; purple, $85-70 \%$; red, $70-55 \%$; yellow, $55-40 \%$; green, $40-25 \%$; and black, $25-10 \%$.

\section{DISCUSSION}

Pulmonary scanning offers a significant addition to diagnostic methods for studying lung function. However, in both our own experience and that of others it provides a relatively non-specific pattern of the pulmonary vascular tree. Thus, similar scan appearances may be found in pulmonary thromboembolism, pneumonia, neoplasm of the lung, and chronic pulmonary disease such as emphysema and pulmonary fibrosis.

MAJOR EMBOLUS The difficulties in interpreting the pulmonary scan with special regard to pulmonary embolic disease are the occurrence of 'false positive' and 'false negative' results. The pattern of the scan in thrombo-embolic lesions is variable. A major pulmonary embolus obstructing blood flow to one lung has an appearance that is almost unique in that the pattern of the scan showing almost total absence of radioactivity on that side is seldom seen in other conditions. The chronic lung conditions, such as emphysematous 

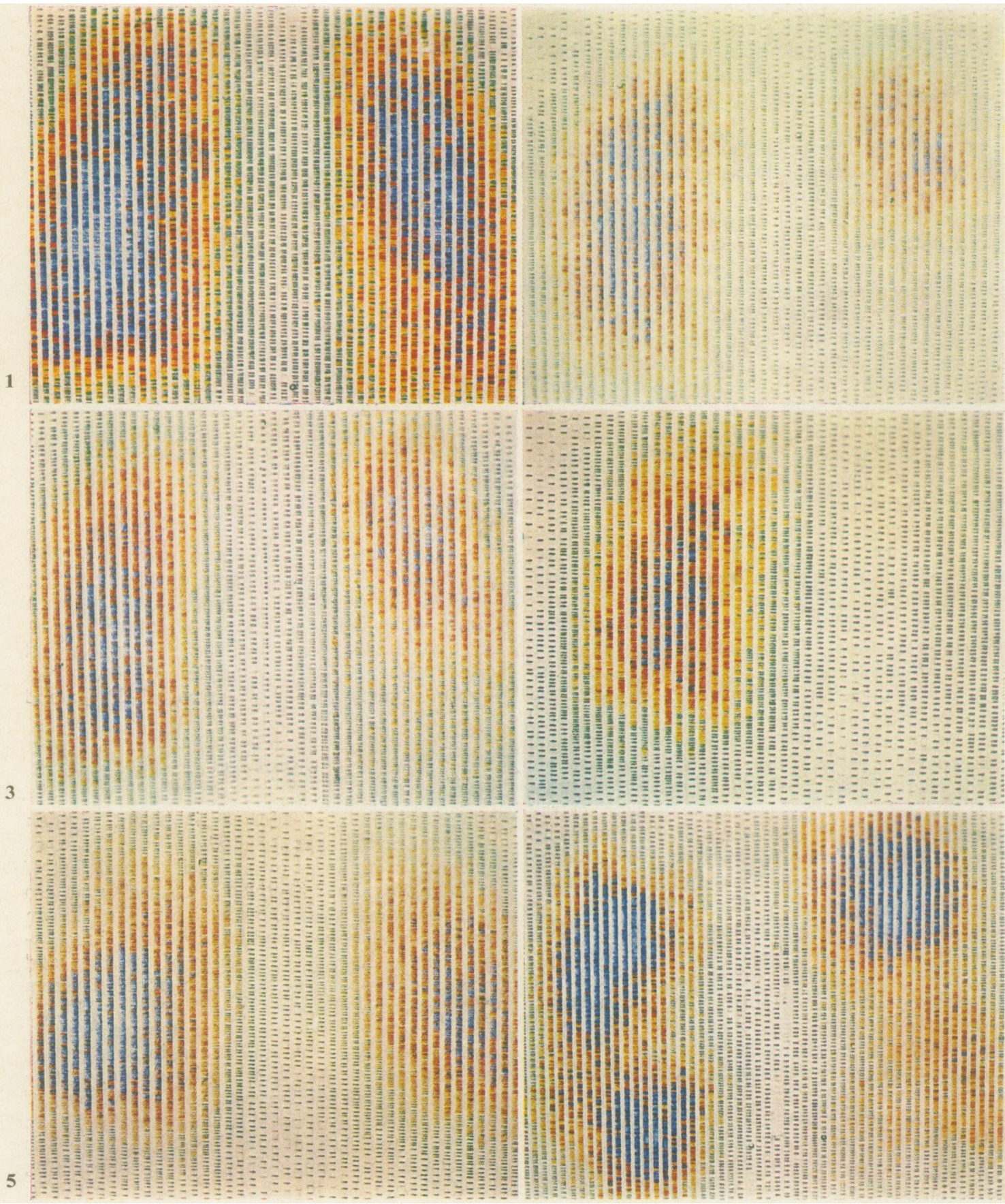

(1) Normal scan. (2) Left basal embolus. (3) Negative scan area at left base, due to cardiomegaly. (4) Major embolus, left pulmonary artery. (5) Bilateral basal emboli. (6) Multiple pulmonary emboli-changes at left base and right mid-zone. 


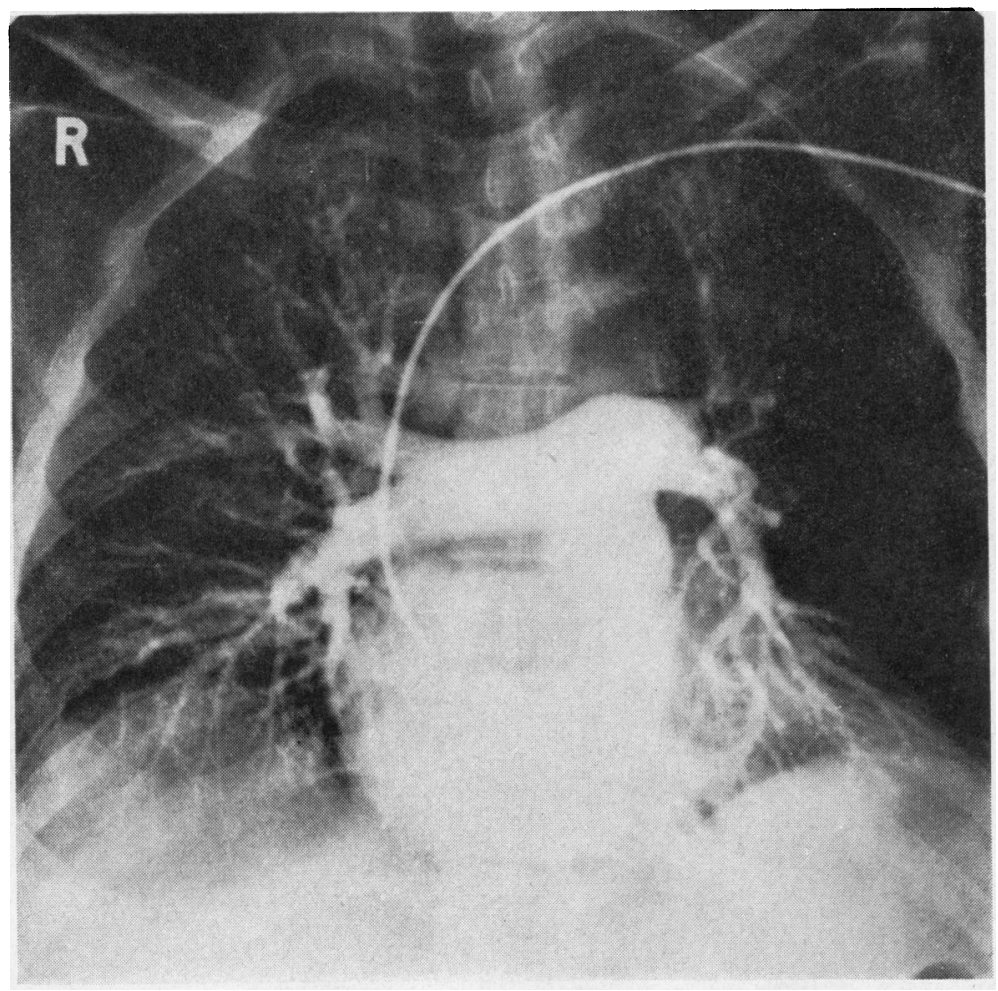

FIG. 1. Pulmonary angiogram demonstrating major left mid-zone embolus.

FIG. 2. Catheter at junction of superior vena cava-main left pulmonary embolus (same patient as in scan 4).

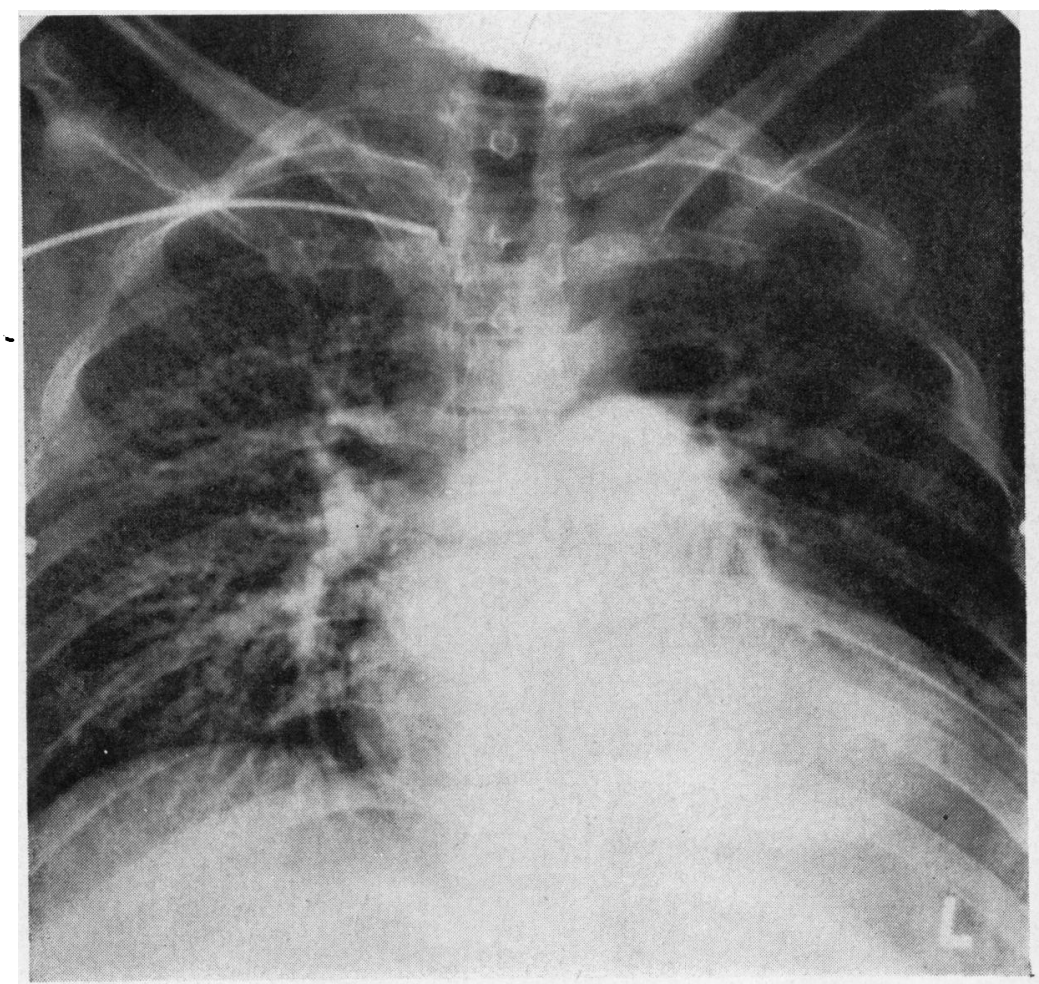




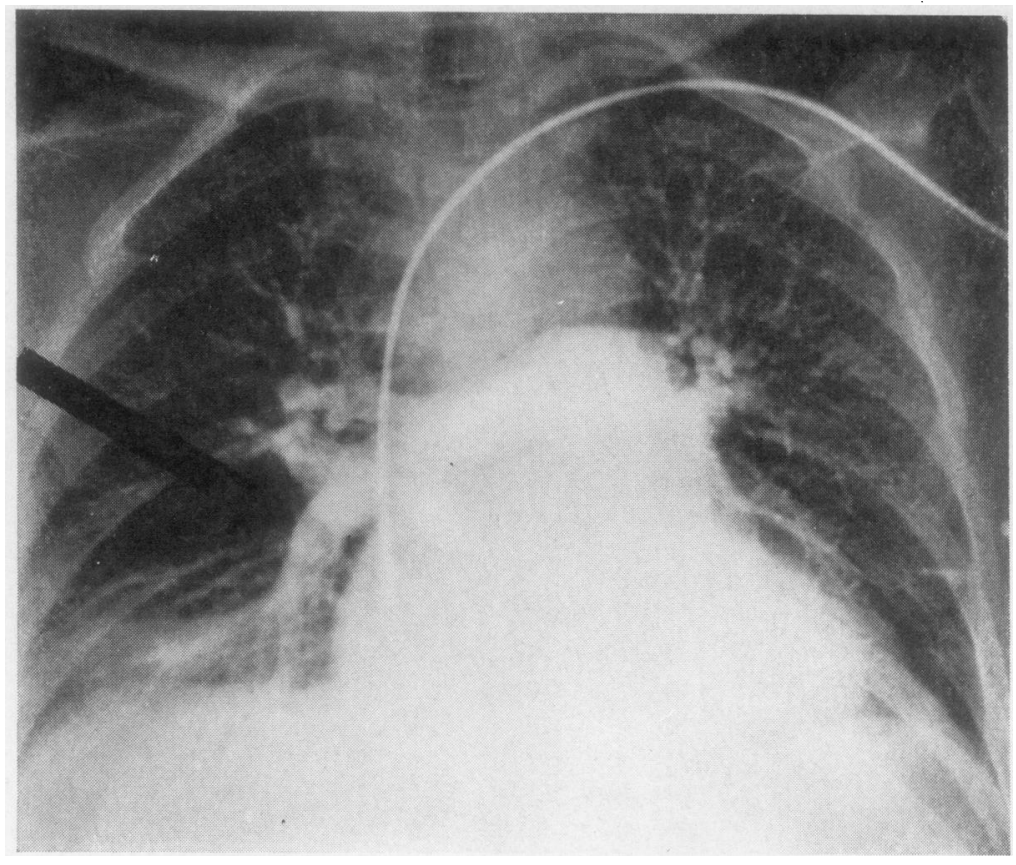

FIG. 3. Embolus right base. Recanalizing at right base.

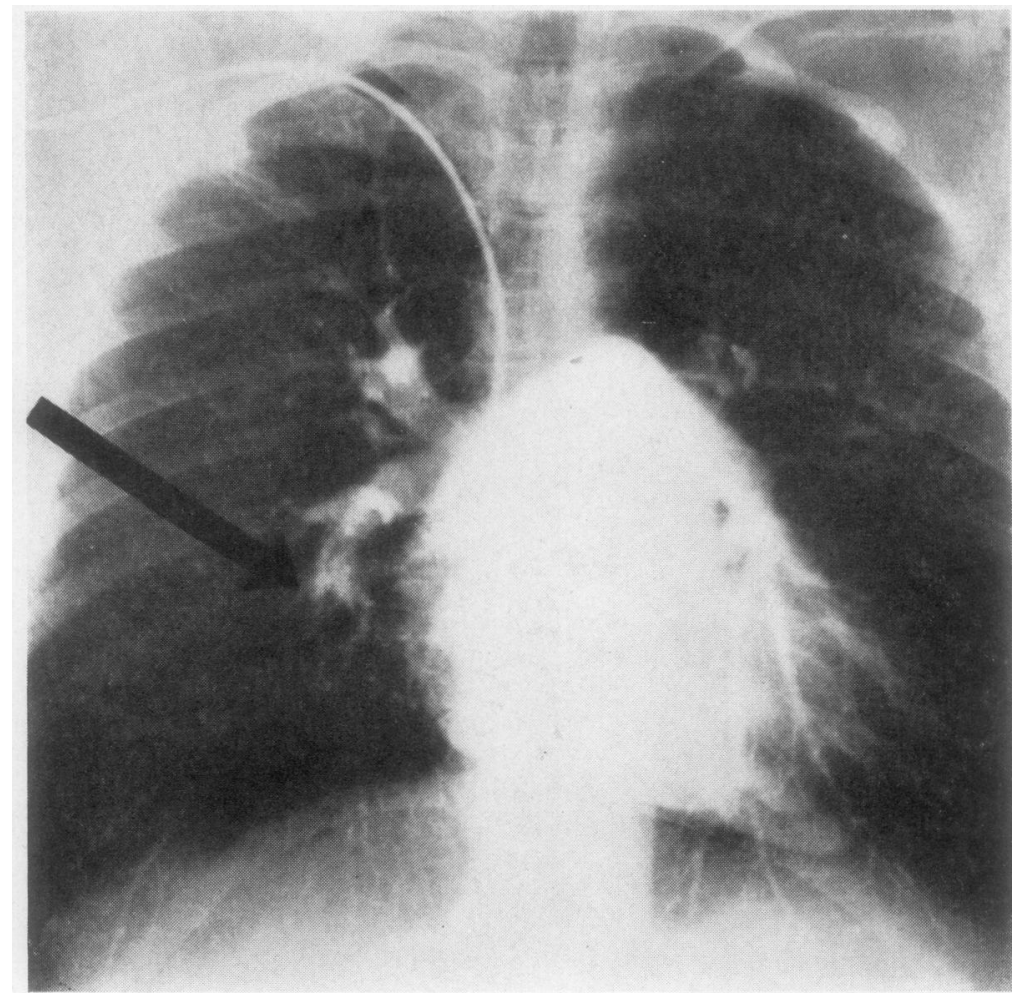

FIG. 4. Corresponds to scan 5. Vessel to right lower lobe occluded (arrow); also avascular zone left costophrenic angle. 
bullae and fibrosis, or acute lesions, such as pneumonia or collapse, have smaller segmental or lobar defects. A hilar neoplasm may produce a similar appearance to massive embolism. However, the clinical history and the patient's condition in these two states are very different, and the routine chest radiograph, though it may raise the question of a prominent hilar lesion being a neoplasm or an enlarged pulmonary artery, is usually distinguishable. It is the general experience that the pulmonary scan will not miss a major pulmonary embolus of this type, and the present series also confirms this. It is worth emphasizing that the case of major pulmonary embolus may be paradoxically the most easily overlooked in diagnosis. Gorham (1961a, b, c) has drawn attention to the various clinical pictures occurring in association with the sudden collapse in a patient with major embolus - the coronary, abdominal catastrophe, and cerebral syndromes. In one patient in the present series, who presented with gross shock and upper abdominal pain, proposed exploration of the abdomen was abandoned on the result of the pulmonary scan which disclosed major bilateral emboli.

EMBOLUS OF LOBAR ARTERY The scanning appearance of a pulmonary embolus lodged in a main lobar artery is quite indistinguishable from that of lobar pneumonia or atelectasis, particularly when, as is usual, the lesion or lesions are at the bases. The majority of pulmonary emboli are bilateral and the right basal zone is predominantly affected. A typical pulmonary scan in such patients shows a general foreshortening of the lung fields, so that on first inspection there appears to be no gross localized defect in the scan and the appearance is only abnormal in the ratio of the length of the lung fields to their width. The situation is comparable to the plain chest radiograph, where the findings may indicate bilateral elevation of the diaphragm without other discernible lesions. (In the first 24 hours a pleural reaction at the costophrenic angle may be absent.)

Though this picture may be regarded as characteristic, the degree of uncertainty with regard to other conditions, for example, post-operative atelectasis, allows of no dogmatism as to the cause of an abnormal szan. One patient in this series suddenly became acutely ill, cyanosed, and collapsed on the fourth post-operative day. The clinical diagnosis of pulmonary embolus seemed certain and a pulmonary scan showed the expected loss of radioactivity at the right base in keeping with the appearance of the plain chest radiograph, which had demonstrated a high right diaphragm and opacification of the right lower zone. However, pulmonary angiography disclosed that the vasculature to this zone was patent and the clinical course suggested that the condition was massive atelectasis of the right lower lobe. Other diagnostically established cases of pneumonia or lobar atelectasis which were studied, however, also showed a pattern of avascularity similar to pulmonary emboli, and it is apparent that the scan is only an adjunct to diagnosis.

SMALL EMBOLI Small pulmonary emboli were not diagnosed by the scan. The problem is mainly the degree of definition by the technique, but improvements in this regard by slowing the scanning speed or always doing a separate anterior, posterior, and lateral scan would be exhausting for ill patients. Simultaneous recording from two focusing collimators (anterior and posterior) with a separate subsequent print-out from a tape recorder of the second detector might offer an advance, but we feel that the technique described in this paper, of simultaneous counting from anterior and posterior tubes, constitutes a considerable improvement over single-tube techniques.

The syndrome of multiple recurrent small pulmonary emboli seems also to escape elucidation by the scanning technique. In the several patients we have studied the scan appeared normal; the widespread nature of the minor arterial zones affected leads to minor symmetrical alterations of blood flow that escape detection. Similarly, in this situation, pulmonary angiography is beset with the diagnostic difficulty that the widespread peripheral pruning of small vessels is almost indistinguishable from alterations in severe bronchitic and emphysematous conditions. Furthermore, in multiple embolic lesions an abnormal scan may occur but the abnormal region may not correspond with the angiographic pattern. The cause of this discrepancy may be functional shunting of pulmonary flow rather than the actual obstruction of vascular structure. With increasing experience of pulmonary scanning it is possible to accept as abnormal scans which show a general shortening in the length (top to bottom) of the lung fields, and it is probable that this is the common pattern in multiple recurrent small emboli rather than the demonstration of delineated zones.

CARCINOMA OF LUNG The value of pulmonary scanning in other conditions requires further 
elucidation. Wagner et al. (1964) have reported the marked avascularity of zones resulting from neoplasm of the lung, and in the present series scans in cases of hilar carcinoma showed absolute avascularity of either an upper zone or an entire lung field. The possibility of diagnostic usefulness is raised by two cases we have studied. A woman had a left hilar and upper mediastinal lesion thought to be either a thymoma or a bronchial neoplasm and showed on pulmonary scanning avascularity of the entire left lung. A correct diagnosis of neoplasm was suggested from the scan result and in this instance a correct prognosis was given to the relatives (bronchoscopy and biopsy had been unhelpful). The second case was a man with partial collapse of the right middle lobe and a possible hilar density. In this instance a pulmonary scan showed little abnormality of the pattern, unlike our previous experience with hilar neoplasm. Following bronchoscopy there was still doubt, and thoracotomy was considered but was postponed for one month and the lesion was found to have resolved.

Atwood, Burchell, and Tauxe (1966) have suggested that the technique of pulmonary scanning is of value in the diagnosis of pulmonary artery agenesis and have reported detection of one case by this means. However, a definitive diagnosis in vascular anomalies such as this will probably continue to be best illustrated by angiographic techniques.

The study of Fred et al. (1966) comprises the most critical analysis of pulmonary scanning in comparison with pulmonary angiography. These workers showed that pulmonary scanning demonstrated pulmonary embolic disease in 26 of 27 instances, but when compared with pulmonary angiography it was less accurate with regard to the location and extent of the lesions in five patients $(19 \%)$. As a result of this finding it was concluded that pulmonary angiography was the definitive and superior technique. Although it must be admitted that this is certainly true, such an assessment needs examination against the background of the practical role of the two techniques and with particular regard to the treatment to be employed in the management of pulmonary embolism. Particular stress was placed by Fred et al. (1966) on the need for angiography prior to surgical embolectomy, and general agreement with this conclusion would be found. However, the present situation with regard to pulmonary embolism is that there is considerable doubt as to how frequently surgical intervention is desirable, since in the majority of cases of even major emboli recovery will follow adequate medical management. In these situations the diagnostic $\stackrel{\mathbb{2}}{\stackrel{2}{2}}$ aid required from pulmonary scanning is to pro-e vide a confirmation of the suspected clinical diag- $\overrightarrow{0}$ nosis, and in this role pulmonary scannning is the safer and less disturbing procedure. Sasahara $\vec{\rho}$ (1967) and several other workers have rebutted in more detail the conclusions drawn by Fred $x$ et al. (1966) in their study.

The pulmonary angiographic techniqueemployed by us has less satisfactory definition than selective angiography, but satisfactoryo opacification was obtained in this series. It has the advantages of being applicable in most radiographic departments and does not require? specialized equipment. Furthermore, patients tolerated the procedure well. It seems that it is a useful compromise between purely intravenous 8 injection into arm catheters (as described by Williams, Wilcox, Andrews, and Burns, 1963) andర selective pulmonary angiography.

We wish to express our thanks to the physicianso and surgeons of the Royal Victoria Hospital. Belfast, 요 for the opportunity to investigate their cases, and to Sister K. Farrell, Mrs. K. Donnelley, and Miss M.Ō Weller for help in this study.

\section{REFERENCES}

Abrams, H. L. (1961). Angiography. Churchill, London.

Atwood, R. M., Burchell, H. B., and Tauxe, W. N. (1966). Pulmonary scans achieved with macroaggregated radioiodinated albumin : use in diagnosis of pulmonary artery agenesis. Amer. J. med. Sci., 252, 84 .

Chrispin, A. R., Goodwin, J. F., and Steiner, R. E. (1963). Th؛ radiology of obliterative pulmonary hypertension and thrombo-? embolism. Brit. J. Radiol., 36, 705.

Fleischner, F. G. (1962). Pulmonary embolism. Clin. Radiol., 13, $169 . \sqsupseteq$

Fred, H. L., Burdine, J. A., Jr., Gonzalez, D. A.., Lockhart, R. W., Peabody, C. A., and Alexander, J. K. (1966). Arteriographic응 assessment of lung scanning in the diagnosis of pulmonary

Gorham, L. W. (1961a). A study of pulmonary embolism. I. A음 clinico-pathological investigation of 100 cases of massive embol-三. ism of the pulmonary artery; diagnosis by physical signs and $\mathrm{N}$ differentiation from acute myocardial infarction. Arch. intern. Med., 108, 8 .

Med., 108, 8. The mechanism of death; based on a clinico- $N$ pathological investigation of 100 cases of massive and 285 cases of minor embolism of the pulmonary artery. Ibid., 108, 189. (1961c). III. The mechanism of pain; based on a clinico- $\sigma$ pathological investigation of 100 cases of minor and 100 cases of massive embolism of the pulmonary artery. Ibid., 108, 418.

McGinn, S., and White, P. D. (1935). Acute cor pulmonale resulting from pulmonary embolism. J. Amer. med. Ass., 104, 1473.

Sasahara, A. A. (1967). Assessment of lung scanning. New Engl. J. S Med., 276, 697.

Schonell, M. E., Crompton, G. K., Forshall, J. M., and Whitby, L. G. T (1966). Failure to differentiate pulmonary infarction from pneumonia by biochemical tests. Brit. med. J., 1, 1146.

Taplin, G. V., Johnson, D. E., Dore, E. K., and Kaplan, H. S. (1964). Suspensions of radioalbumin aggregates for photoscanning the liver, spleen, lung and other organs. J. nucl. Med., 5, 259.

Tow, D. E., and Wagner, H. N., Jr. (1967). Recovery of pulmonary arterial blood flow in patients with pulmonary embolism. New Engl.J. Med., 276, 1053.

Wacker, W. E. C., and Snodgrass, P. J. (1960). Serum LDH activity in pulmonary embolism diagnosis. J. Amer. med Ass., 174, 2142응

Wagner, H. N., Jr., Sabiston, D. C., Jr., McAfee, J. G., Tow, D. and Stern, H. S. (1964). Diagnosis of massive pulmonary embolism in man by radioisotope scanning. New Engl.J. Med., 271, 3770

Williams, J. R., Wilcox, W. C., Andrews, G. J., and Burns, R. R. (1963). Angiography in pulmonary embolism. J. Amer. med.
Ass., 184, 473 . 